\title{
La transformación del complejo minero Zollverein: un híbrido entre la ciudad difusa y lineal.
}

\author{
Rene Carlos Davids Chambre \\ Departamento de Arquitectura, University of California, Berkeley, California, USA \\ E-mail: rdavids@berkeley.edu
}

\begin{abstract}
Resumen. El aumento del desarrollo de las fuentes de energía renovable y las importaciones de bajo costo han eliminado la necesidad de la producción alemana de carbón. En 1986 la minería en el complejo Zollverein de Essen, Alemania que fuera en 1932 uno de los más avanzados del mundo, había cesado de funcionar. Para promover la renovación urbana y ecológica de las antiguas zonas industriales y mineras contaminadas, el gobierno estatal de Renania y Westfalia del Norte- lanzó en 1989 un concurso (Internazional Bauaustellung $B A)$, que desarrolló una política progresista de conservación y restauración y un programa de paisajismo de bajo mantenimiento. Junto a los edificios industriales modernistas los esfuerzos de IBA le aseguraron al complejo Zollverein un lugar en la lista del patrimonio mundial de la humanidad (UNESCO 2001). A pesar de que el trabajo ha sido ampliamente publicado y halagado por los esfuerzos de conservación, las lectura urbanas del conjunto no ha llamado mayormente la atención. Esta ponencia postula que al unir a través un bulevar (que solía ser una línea férrea) trés nucleos urbanos de alta densidad rodeados de paisaje, como una especie de puente uniendo las islas de un archipiélago, el complejo Zollverein actual presenta una propuesta de gran interés urbano; al proponer núcleos densos en forma discontinua y rodeados por paisaje, el excentro minero sugiere un propuesta hibrida entre ciudad lineal tradicional y una entidad difusa y extendida.
\end{abstract}

Palabras clave: urbanismo lineal, urbanismo difuso, paisajismo, energía, ambiental, parque, sostenibilidad.

\section{Introducción}

El complejo industrial de la mina de carbón de Zollverein establecida en 1847 en Essen Alemania pasó a ser en el siglo XX la más grande, de mayor rendimiento y más moderna de Europa. El nombre "Zollverein" se refiere al acuerdo de una unión aduanera firmado en 1834 por una coalición de estados alemanes liderada por Prusia para implementar políticas económicas que favorecían el intercambio comercial e industrial. A raíz de ese acuerdo se inició a mediados del siglo XIX, un período de industrialización continua en Ruhrgebeit que se extendió hasta bien entrada la segunda mitad del siglo XX. A lo largo de gran parte del siglo, la industria del carbón y el acero impulsada primero por las dos guerras mundiales y posteriormente por la reconstrucción de la posguerra. convirtió a esa región en la más rica de Alemania. Representando el auge quizás mejor que otros complejos mineros, el Zollverein diseñado en 1932 por Fritz Schupp y Martin Kremmer en el estilo de Neue Sachlichkeit o "nueva objetividad" al demostrar una síntesis de nuevas formas a las cuales le había dado fama la escuela Bauhaus y tecnologías avanzadas, fue desde un principio un conjunto importante y ha pasado hoy en día a ser considerada una obra arquitectónica maestra. (Fig. 1)

En 1961, se agregó al conjunto Zollverein diseñado también por Schupp y Kremer, una nueva planta de coquización utilizando el 


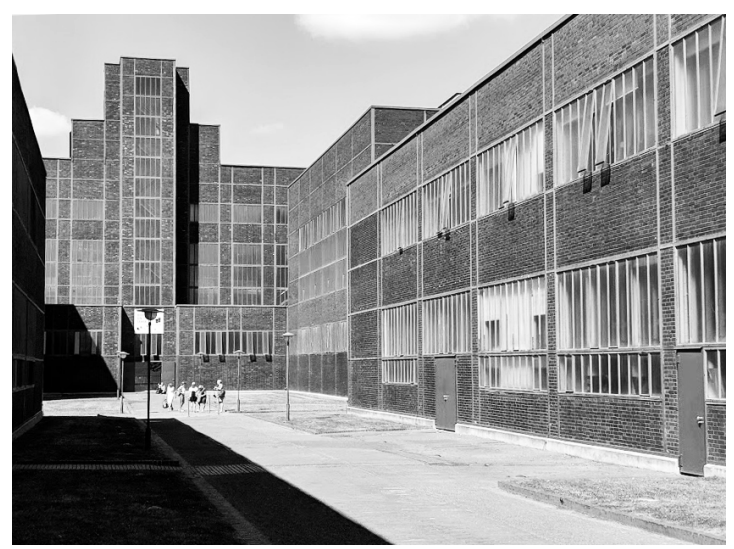

Fig. 1. Zollverein. Casa de la Caldera. Foto RD

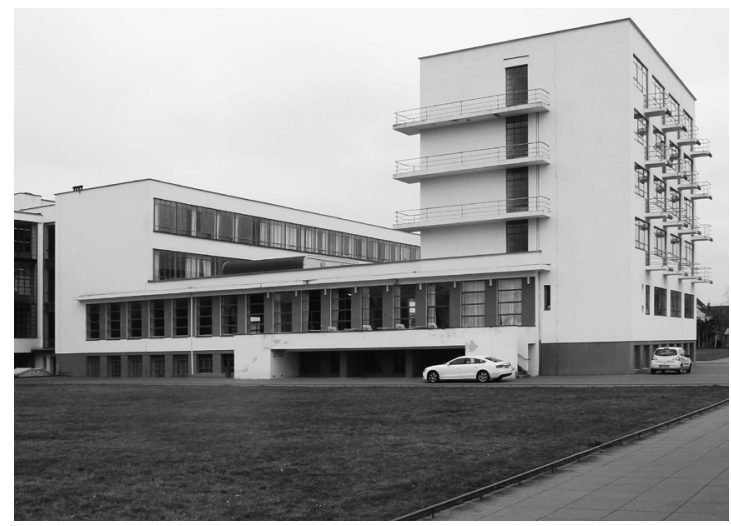

Fig. 2. Bauhaus. Foto RD

mismo vocabulario arquitectónico: armazón de acero ortogonal, ladrillo rojo y paneles de relleno de hormigón ${ }^{1}$. A pesar de que muchas de las ventanas fuesen diseñadas de acuerdo a la preferencia modernista por las bandas horizontales otras, al establecer líneas de composición vertical y simétricas cómo en la sala de calderas en el complejo del Pozo XII reforzaban, la tradición clásica que también se acentuaba con el uso del ladrillo, el material tradicional de la región del Ruhr de Alemania. Además de usar ventanas horizontales y verticales, los arquitectos también usaron grandes ventanales con el objeto de revelar la ubicación de máquinas tales como compresores o de transporte. El complejo Zollverein al ser un híbrido entre lo clásico y lo moderno por lo tanto se diferencia de edificios contemporáneos modernistas como el Bauhaus diseñado por Walter Gropius en Dessau de 1925 a 1926 que es una edificio formal, abstracto y blanco, que se basa en un planimetría dinámica y asimétrica similar a un remolino. (Fig. 2)
Estilísticamente híbrido, el complejo de Zollverein fue apropiado por dos movimientos radicalmente opuestos: para los Nazis el Zollverein fue un excelente ejemplo de arquitectura industrial porque no se parecía a los edificios que ellos atribuían a los bolcheviques mientras que para la generación de la postguerra pasó a ser un ejemplar de la arquitectura de la república democrática Alemana de Weimar. En realidad, el Zollverein, probablemente debería considerarse como un producto de lo que Jeffrey Herf llamó la compatibilización o conciliación entre tecnología y la falta de raciocinio o la mezcla de modernidad combinada con los sueños del pasado; una ideología que comenzó en las universidades técnicas alemanas a principios de siglo y terminó siendo adoptada por el partido $\mathrm{Nazi}^{2}$.

Junto al interés en una versión regionalista y semiclásica del estilo internacional, Schlapp y Kremmer también exploraron técnicas avanzadas de construcción cómo por ejemplo la soldadura de arco. A pesar de que se conocía desde fines del siglo XIX y fuera patentada en 1887 , la soldadura de arco no resistía construcciones con estrés dinámico, pero la técnica se perfeccionó y comenzó a popularizarse debido al conocimiento adquirido con la construcción de aviones durante la Primera Guerra Mundial. A raíz de ese avance, las vigas sólidas de banda ancha reemplazaron aquellas de miembros delgados cruzados equis prevalentes antes de los años 20. Las vigas de banda sólida eran más livianas y se podían curvar, permitiendo fabricar formas imposibles de obtener can las tradicionales vigas doble $\mathrm{T}$. El desarrollo de las estructuras de acero constituyó un importante paso en la promoción la tecnología de soldadura de arco, pero fue más que nada la combinación de muros de cortina con las vigas de bandas sólidas que establecieron a los edificios del Zollverein como vanguardistas ${ }^{3}$. Esto, a pesar de que, a diferencia de otras fábricas como Boots en Nottingham del ingeniero Sir E. Owen Williams en 1930 y la fábrica Nelle de Brinkman \& Van der Vlugt de1928 en Rotterdam que también utilizaran muros-cortinas, ambas el complejo Zollverein fuera diseñado con muros de ladrillos en vez del leguaje abstracto modernista. Además de 
utilizar nuevos métodos de construcción, el complejo Zollverein también empleó avances tecnológicos que permitieron una aceleración y racionalización considerable del proceso minero, tal como el uso de locomotoras eléctricas subterráneas que transportan carbón a largas distancias hasta un foso central.

\section{Renacimiento}

Los efectos combinados del aumento de los costos de producción nacional, la disminución del precio del carbón importado, el bajo precio del gas natural y el aumento de la disponibilidad de energía provenientes de recursos renovables hicieron que la industria minera alemana de carbón perdiera competitividad y en 2013, la producción había disminuido en casi un $90 \%$ desde los niveles máximos alcanzados varias décadas anteriores ${ }^{4}$.

Para promover la renovación urbana $\mathrm{y}$ ecológica en las antiguas zonas industriales y mineras de carbón altamente contaminadas del Ruhr, que incluían diecisiete ciudades, el gobierno del estado federado de Renania del Norte-Westphalia implementó, en 1989, la Internationale Bauaustellung, IBA (Exposición Internacional de Construcción), un importante programa de restauración. que en la década siguiente organizó talleres, concursos internacionales de diseño y sesiones de planificación pública, para estimular la participación ciudadana en la creación de más de 100 proyectos en cinco sitios separados ubicados dentro de los 800 kilómetros cuadrados que separan las ciudades. de Duisburg y Kamen ubicadas en márgenes opuestos del Ruhr ${ }^{5}$. Al reutilizar y conectar diversos fragmentos de paisaje, muchos de ellos remanentes de la industria minera, pero que también incluían tierras agrícolas, bosques, y vegetación en terraplenes ferroviarios, el IBA creó acceso continuo de espacios verdes para el público a través de la zona. Una gran cantidad de líneas ferroviarias desaparecidas dentro de los límites del parque que previamente habían prestado servicio a las minas se transformaron con el apoyo de la IBA y financiamiento público, en una red regional de senderos para ciclistas y peatones.

La iniciativa Emscher Landscape Park se basó en el concepto de Industriekultur, (cultura industrial) que vincula la conservación histórica y educación con la recreación, y "Industrienatur" (naturaleza industrial) una práctica de paisaje que tenía como objeto fomentar la restauración espontánea de comunidades de plantas nativas que requieren bajo esfuerzo de mantenimiento. (Fig. 3) Al reconstruir el paisaje natural como pudo haber sido antes del establecimiento de las industrias se crea una relación armoniosa entre las ruinas industriales y el, creando una estética de carácter único del Ruhr ${ }^{6}$. Al trabajar con el paisaje existente en lugar de remodelarlo como se había hecho durante siglos, (1994) el parque

Fig. 3. Duisburg Nord 2018 Foto RD

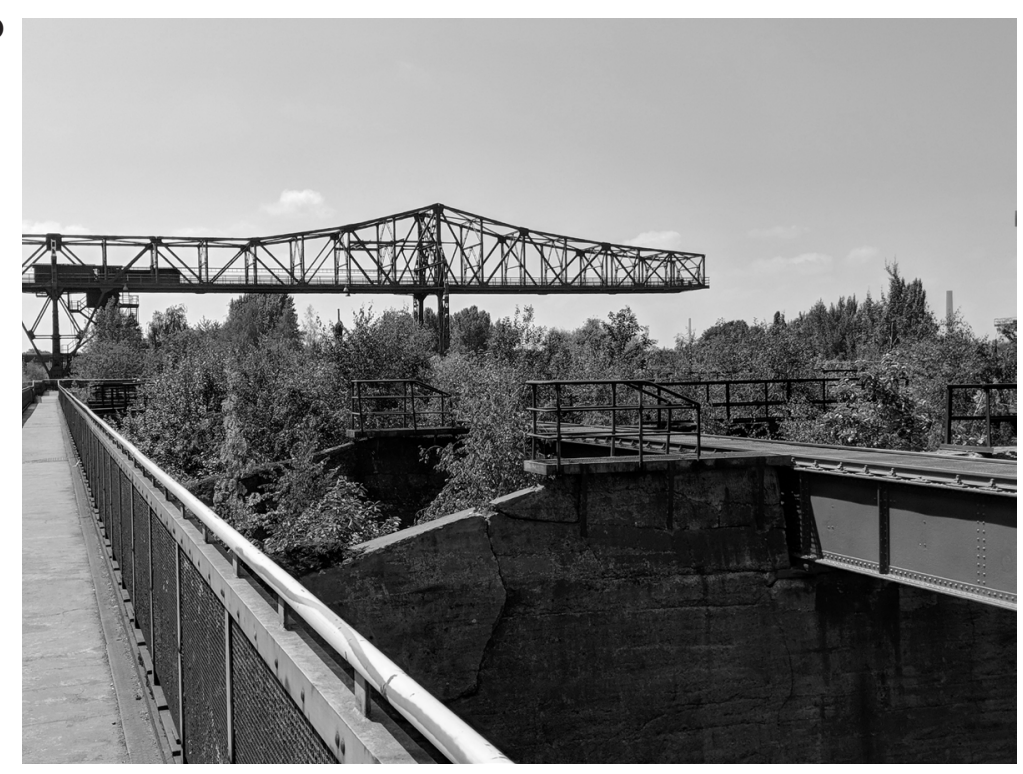


de Duisburg Nord diseñado por el arquitecto paisajista PeterLatz, que fuera la transformación de la fundición de Thyssen Hochofenwerk Meiderich fue la primera industria en que se aplicó la Industrienatur cuando dejó de operar en 1985. Peter Latz reestructuró el diseño de Duisbug Nord a través de cuatro categorías: "Parques de Hornos","Parques de Rieles", "Parques Acuáticos", que incluye canales y cuencas de captación, y "Parques de Vegetación" que combina jardines pequeños y de gran escala con los edificios industriales existentes. El diseño del paisaje de Duisburg Nord fue influyente no sólo en la región del Ruhr sino también a través del mundo ${ }^{78}$.

La otra transformación importante en el Ruhr comenzó, despues de 1993 cuando cerró el Zollverein, la última mina de carbón en funcionamientodela ciudaddeEssen. En2001 se contrató (Office of Metropolitan Architecture) la oficina OMA con sede en Rotterdam para que estableciera pautas económicas y creasen un plan maestro de desarrollo para transformer el complejo Zollverein en un parque cultural y empresarial. Los diseñadores dividieron el complejo en cuadrantes: los tuneles 1,2 y 8 , el Eje XII, la planta de coque y el bosque de esculturas. El paisaje fue diseñado en 2003 por Agence Terre, de París, e implementado por los arquitectos paisajistas alemanes: Planergruppe $\mathrm{GmbH}$ de Oberhausen ${ }^{9}{ }^{10}$. OMA tuvo como objetivo preservar la mayor cantidad posible de edificios para asegurar el listado del complejo como monumento del "Patrimonio Mundial de la Humanidad" y, en 2001, se reconoció efectivamente como un ejemplo sobresaliente de la arquitectura moderna con una importante colección de edificios industriales europeos para la extracción y el tratamiento del carbón y la producción de coque, incluyendo la red de líneas ferroviarias de servicio y los vastos desechos contenidos en su entorno. Hoy en día el parque contiene importantes instalaciones culturales que incluye la antigua casa de calderas rediseñada por el arquitecto Norman Foster en un centro de diseño que también alberga el "Red Dot Design Museum" con la colección de diseño contemporáneo más grande del mundo y el Museo del Ruhr, rediseñado por OMA en la antigua planta de lavado de carbón que relata la historia de la zona. El complejo también contiene la Escuela de Gestión y Diseño Zollverein, en el Cubo ("Sanaa Cube") diseñado por la oficina japonesa de arquitectura, Sanaa. (Fig. 4)

\section{Memoria}

La estructura del foso XII de la mina Zollverein pasó a ser un símbolo de la transformación del Ruhr de una region industrial que se caracterizaba por la producción de carbón y acero en una economía basada en creatividad tecnológica y educación (Fig. 5). El Ruhr era una region que no contaba con instituciones educativas pero se enorgullece hoy de las cinco universidades.de la zona. Paradojalmente, a pesar de que la preservación de las estructuras industriales del Ruhr ha pasado a ser una parte muy importante de la nueva identidad de la región, la historia misma de las vidas de los trabajadores en el contexto socio-económico y politico de la época, pasa desapercibida. A diferencia de los documentos escritos, los edificios contienen una rica realidad física que es independiente de la información histórica
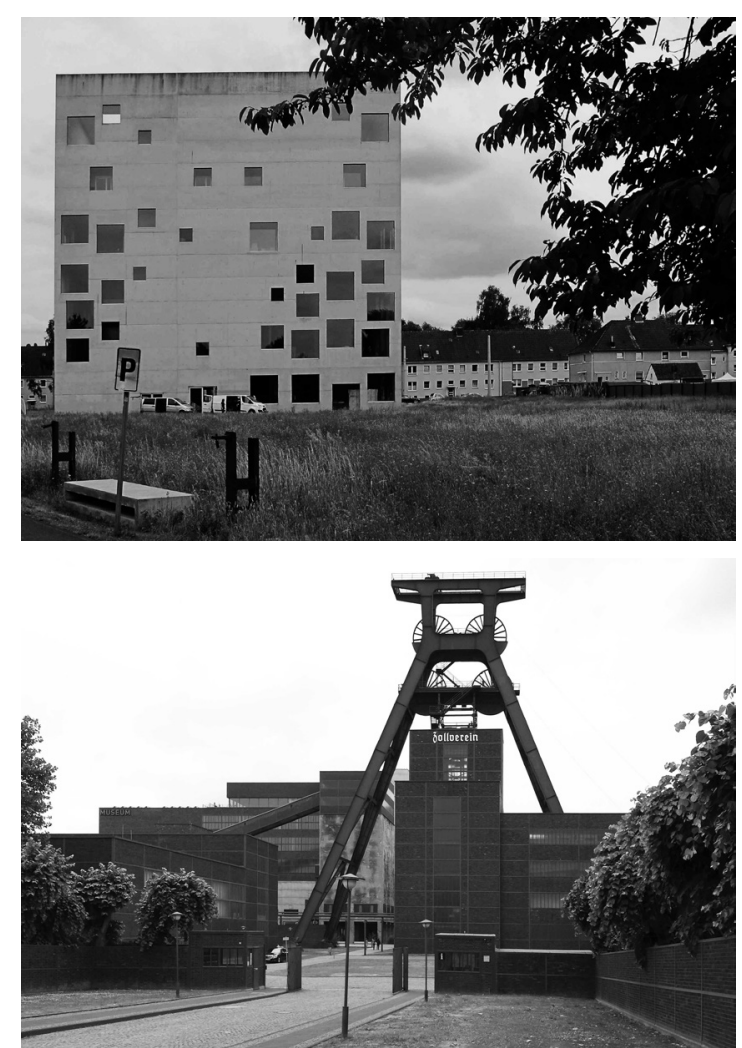

Fig. 4. (arriba) Sanaa Zollverein. Foto RD

Fig. 5. (abajo) Zollverein. Foso XII. Foto RD 
que proporcionan. Las ruinas en especial generan una misteriosa lectura poética que permite ignorar el hecho de que la industria del carbón y del acero proporcionaran una vida decente, aunque a menudo dura, incluso brutal y peligrosa para millones de trabajadores. La ciudad de Essen también fue clave para el rearme alemán del Tercer Reich y el conglomerado Krupp radicado allí saqueó y explotó recursos, propiedad privada y pública de los países ocupados y e utilizó escalvos de los campos de concentración en sus actividades productivas. A pesar de que las ruinas frecuentemente oscurecen el pasado continuamos insistiendo en atribuírles el poder de transmitir nuestro legado cultural. A diferencia del occidente, en Japón, la concepción del patrimonio está ligada a la creencia de que la eternidad reside en su capacidad para sostener el cambio, como lo demuestra la reconstrucción periódica del Santuario Ise y la reubicación de edificios históricos en la aldea Meiji ${ }^{11}$. De manera similar, el valor de las ruinas para el autor Sebastien Marot no radica tanto en preservar las propiedades culturales del pasado como en dar forma a la cultura contemporánea, al permitir la participación de un sitio, a través de múltiples lecturas ambivalentes; la memoria se entiende entonces en vez de ser un depósito de recuerdos, cómo un acontecimiento evolutivo que expande la percepción de nuestro entorno espacio-temporal ${ }^{12}{ }^{13}$. Los recuerdos de cada época de nuestra vida se conservan entonces pero, a diferencia de los restos óseos fósiles que permiten el reconocimiento de las entidades de las que alguna vez fueron parte; son más bien cómo las piedras de ciertas casas romanas que provienen de edificios cuya su antigüedad no puede establecerse por su forma o su apariencia, sino solo a través de sus rasgos.

El otro factor que oscurece las narrativas del pasado socio-económico del Zollverein es una reacción estética o sentimiento que entendemos cómo lo sublime y que se vincula a las industrias del siglo XIX y principios del XX con las cuales el capitalismo parecía dominar la naturaleza. Desde el siglo XVII en adelante, lo sublime se asoció con emociones que sugieren lo enigmático un temor, la inmensidad, el infinito, el vacío, la soledad y el silencio. Transmitido por escritores y pintores particularmente en relación con el paisaje natural, lo sublime también crea una especie de estado a-histórico, un grado de suspensión entre el pasado y el presente. Hoy en día lo sublime también se asocia con obras como las instalaciones del artista James Turrell, que evocan inmersión una inmensidad espacial ${ }^{14}$ ${ }^{15}$. Dentro de la arquitectura contemporánea, el trabajo del arquitecto Daniel Liebeskind ha sido identificado a través del diseño para el Museo Judío de Berlín, al apuntar hacia algo mucho mayor, elegíaco y pertubador, con lo sublime. Con su mezcla de estructuras monumentales a veces rehabilitadas y otras veces oxidadas, frecuentemente rodeadas de vegetación nativa el complejo Zollverein también evoca lo sublime ${ }^{16}$. Peter Latz, de vé en las ruinas industriales abandonadas del Ruhr una experiencia simultáneamente placentera y amenazadora, semejante a la provocada por las grotescas esculturas que se encuentran dispersas dentro de los límites del Parco dei Mostri, (el parque de los monstruos), diseñado por el arquitecto Pirro Ligorio en $1552^{17}$ (Fig. 6). Dada su monumentalidad y relación con la naturaleza los edificios del Zollverein también recuerdan a conjuntos prehispánicos como, por ejemplo, las pirámides de Teotihuacán, sin llevar por supuesto, la carga simbólica. Al producir una sensación de misterio e incluso

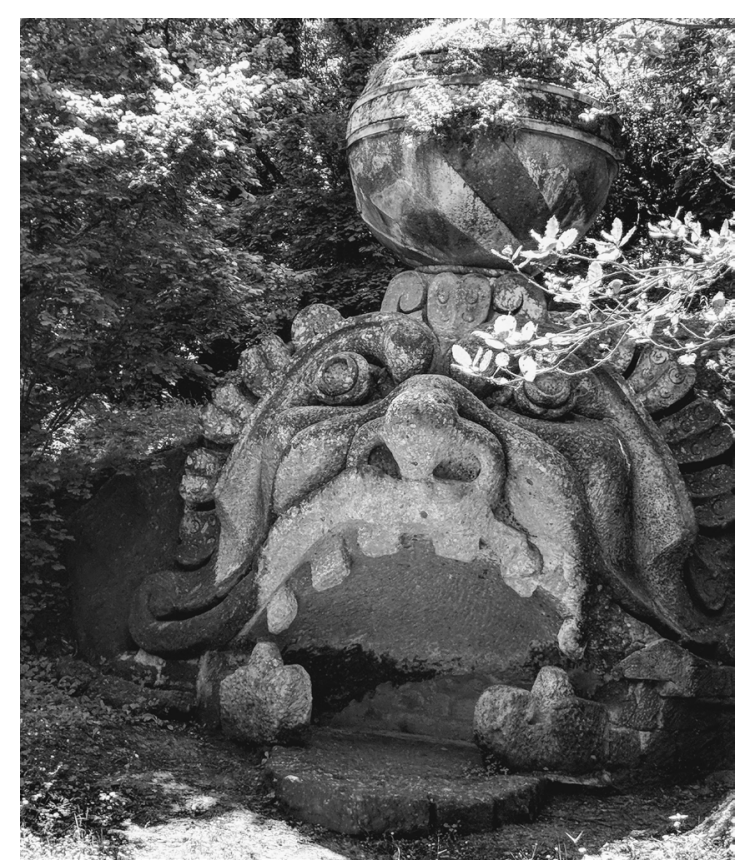

Fig. 6.Parco di Mostri. Foto RD 
de temor los edificios del complejo Zollverein y contrastan con la claridad cristalina de los edificios del Plan Voisin o la tranquilidad generada por las alusiones históricas que caracterizan los complejos diseñados a lo largo de los preceptos del neo -tradicionalismo.

\section{Nueva urbanidad}

Originalmente organizados por la lógica de la producción y el transporte de carbón, los edificios de Zollverein se utilizan hoy en día para la recreación y la cultura; las plantas industriales se han convertido en museos, los montículos de desechos en montículos dentro de los jardines y las conexiones ferroviarias en senderos o "bulevares". La transformacón del Zollverein en general ha sido dramática, pero las calles y las viviendas de trabajadores de los alrededores, no han sufrido cambio ${ }^{18}$. Por otro lado, algunos elementos de la industria no han han dejado de funcionar pero permanecen formalmente intactos. Las grandes chimeneas que solían contaminar el aire por ejemplo ahora apuntan a cielos limpios, y recuerdan los obeliscos, los capiteles de iglesias o las cúpulas de la ciudad barroca, que establecían una connection entre la tierra y el cielo.

Las chimeneas contrastan con el sentido horizontal de las vías férreas que parecen extenderse hasta el infinito ${ }^{19}$. Debido a su centralidad en el complejo, la vía férrea más importante es el Track Boulevard, un sendero para bicicletas y peatones que, a diferencia de su clásico homónimo francés, no esta confinado regularmente por un tejido residencial sino que, sobre todo, lo está por la vegetación nativa de densidad variada (Industrienatur) y solo ocasionalmente por edificios ${ }^{20}$ (Fig. 7). El Track Boulevard es parecido a las redes de senderos que han surgido en líneas de ferrocarril abandonadas pero la preservación de los rieles en el pavimento ayuda a proporcionar a este ultimo un carácter más industrial y urbano. (Fig. 8)

En contraste con la High Line, otra línea ferroviaria transformada pero de extensión limitada, el bulevar se ubica a nivel de suelo y podría por lo tanto extenderse y que la naturaleza industrial (Industrienatur) podría fácilmente establecer los bordes a sus costados.
El Track-Boulevard se puede comparar a Allée, un sendero típico del jardín formal francés que era un paseo que parecía extenderse hasta el infinito y normalmente pasaba a través de un pequeño bosque recortado de forma rectangular. Sin embargo, en el Zollverein en lugar de un episodio discreto, el bosque es como un sólido al cuál se le extraen espacios y caminos, equivalente a un tejido urbano, pero a diferencia de éste último está formado por vegetación. Desde éste punto de vista, el complejo Zollverein no es un parque con edificios, sino una ciudad formada simultáneamente de edificios y materia verde. Al igual que algunos centros urbanos en Europa y EE.UU y con la excepción de ser recorrido por dos calles vehiculares; el complejo Zollverein segrega el movimiento peatonal en el interior del tráfico en su periferia, dejando una gran área verde en el centro. A diferencia y del esquema de Radburn en Nueva Jersey de1929, o Baldwin Hills Village de 1950 (que ahora se
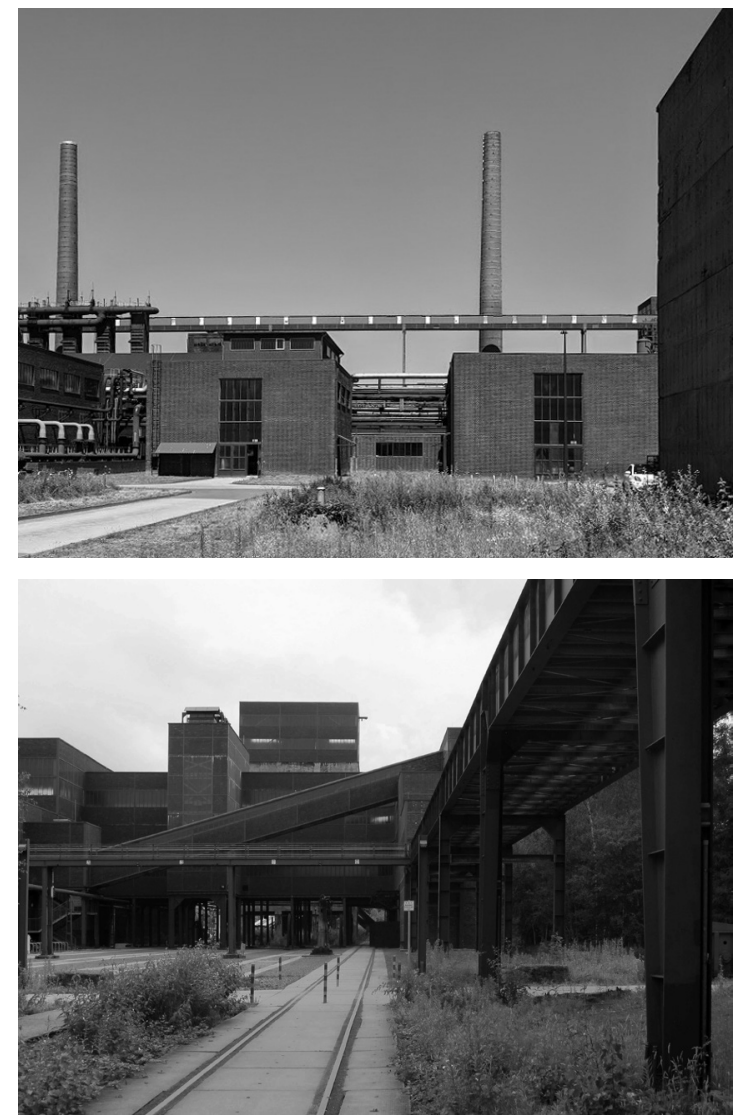

Fig. 7. (arriba) Zollverein. Foto RD

Fig. 8. (abajo) Track Boulevard. Zollverein. Foto RD 
llama the Village Green) ambos diseñados por los planificadores Henry Wright y Clarence Stein que también segregan los movimientos y dejan espacios verdes en el centro en el cúal pueden jugar los niños o descansar los adultos, en el del complejo Zollverein el área central lo ocupa un bosque nativo llamado Bosque de Esculturas (Skulpturenwald) que no es un área de reunión o de juego sino que un espacio de contemplación de esculturas que ayuda a realzar los espacios de alrededor. De hecho, el bosque está rodeado en su periferia por tres complejos; la planta de coque, el Foso de mina XII y el foso de mina 1/2/8 que forman una especie de archipiélago de islas urbanas rodeadas por la naturaleza industrial (Industrienatur). Los edificios Zollverein al estar separados unos de otros se podrían comparar a la planificación del Plan Voisin de Le Corbusier de 1925 para París, pero a diferencia de éste último, que disponía de torres cristalinas ampliamente espaciados, los edificios no son trasparentes, se relacionan estrechamente entre sí y en general están rodeados por una densa naturaleza industrial (Industrienatur) a diferencia de un parque.

La disposición de los edificios en el complejo Zollverein que llamamos Planificación Industrial (Industrieplanung) presenta, una organización formal de un archipiélago con islas de edificios rodeados por la naturaleza Industrial (Industrienatur) y unidos por senderos y bulevares. Si bien el complejo Zollverein no incluye viviendas dentro de sus límites, desarrollos futuros basados en ella podrían incorporarlos fácilmente. El conjunto industrial recuerda el archipiélago verde the Matias Ungers, que fuera una propuesta para tratar de resolver los problemas que enfrentaba la ciudad de Berlin cuando había perdido población y todavía contenía escombros y ruinas visibles de la seguda guerra mundial. y estaba rodeada por la República Democrática de Alemania. Ungers consideraba la situación no tanto un problem a resolver sino que cómo una oportunidad para desarrollar una propuesta; la idea consistía en establecer una serie de centros consolidados o islas alrededor de edificios que estaban en buen estado y demoler las zonas de la ciudad que habían sido abandonadas o que estaban en un proceso de deterioro. En estas últimas, Ungers proponia establecer un sistema de agricultura que les permitiría a los habitants crecer sus propias verduras así mismo como zonas de recreación $\mathrm{y}$ viviendas de baja densidad ${ }^{21}$.

La idea del archipiélago rodeado de un mar verde contenido en el complejo de Zollverein y presenta un gran potencial cómo ejemplo para la creación de comunidades nucleares a lo largo de líneas de ferrocarril abandonadas u otros corredore lineales combinando características de la ciudad policéntrica y tradicional. A diferencia de la idea del archipiélago de Ungers el Zollverein sugiere que las areas alrededor de las islas serían de vegetación nativa densa, la llamada Industrienatur.Al igual que ciudad lineal concebida por Arturo Soria y Mata. publicada en 1882, el Zollverein sugiere una posible extension del complejo a lo largo de una vía de circulación bordeada.. El Zollverein sugiere que zonas urbanas pordrían surgir a lo largo de la via (Track boulevard) en forma de islas o núcleos como un collar de perlas interceptado por la industrienatur. (Fig. 9 y Fig. 10)

\section{Conclusión}

Cuando se inauguró en 1932, el Zollverein en Essen, diseñado por el despacho de arquitectura Schupp \& Kremmer fue considerado el complejo minero más grande moderno y avanzado de Europa y la "mina más hermosa" del mundo". Hoy, después de haber dejado

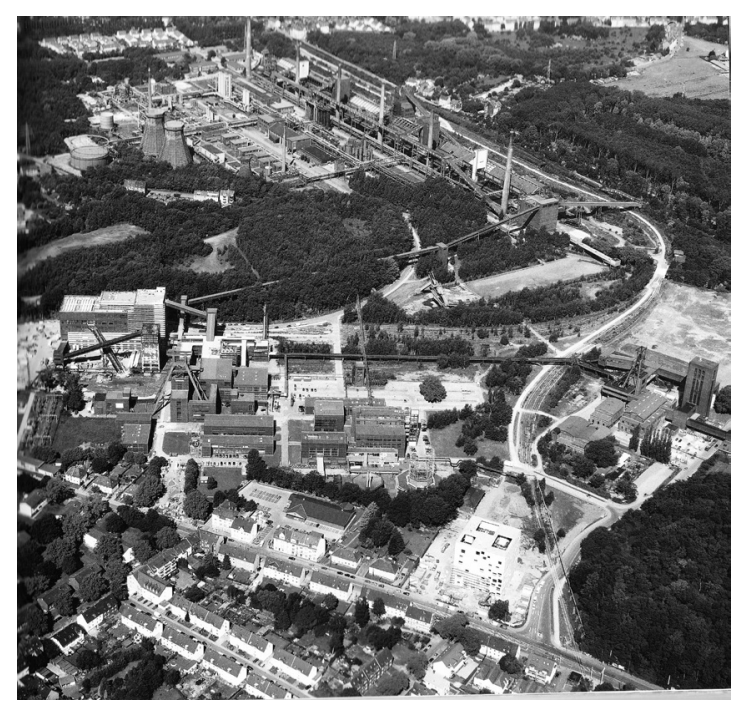

Fig. 9. Zollverein. Foto aérea 


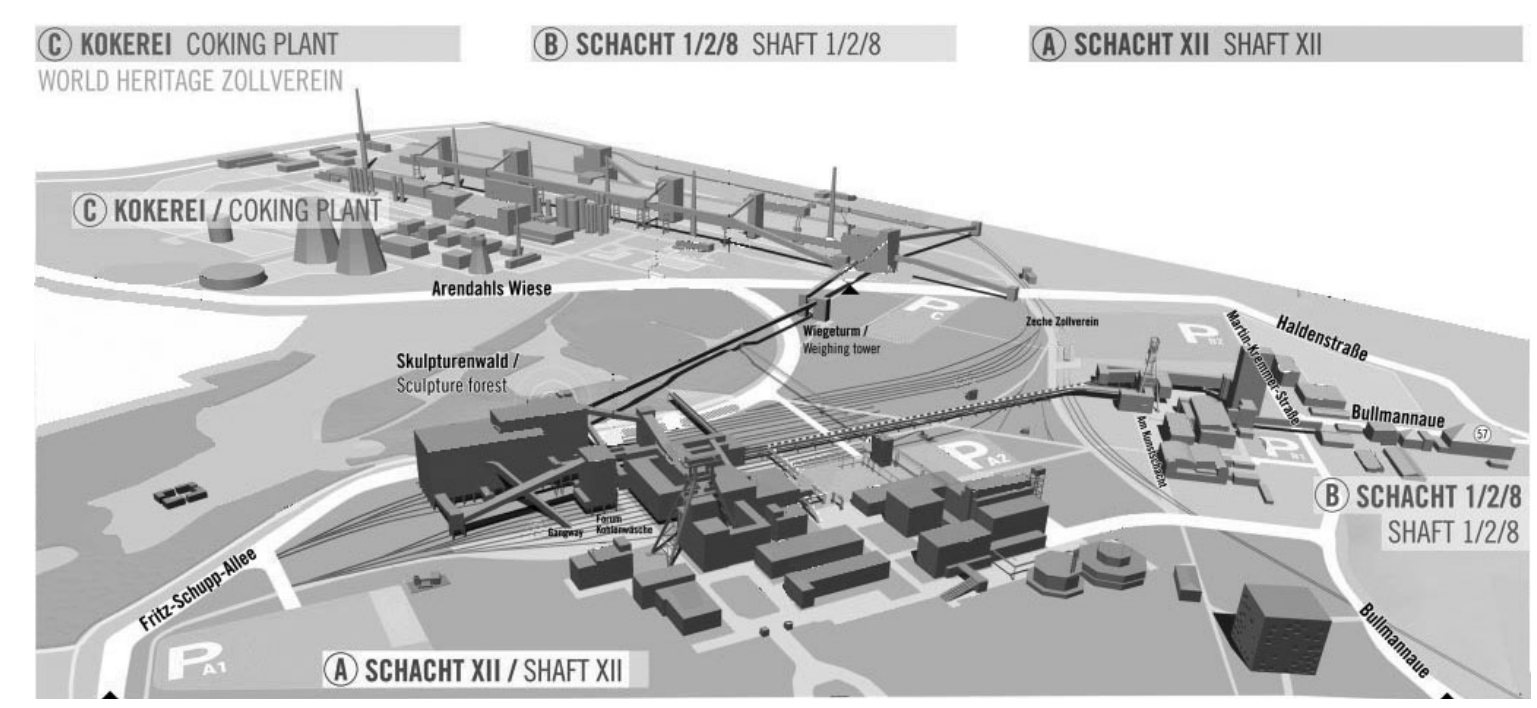

Fig. 10. Zollverein. Tres áreas

de operar en 1993, el conjunto Zollverein, al igual que el parque Duisburg Nord es admirado por su paisajismo de bajo mantenimiento y la conservación y la reutilización de sus edificios. Al mismo tiempo el Zollverein también ha introducido nuevas tecnologías como por ejemplo el uso de la casi inagotable energía geotérmica que emite una cantidad mínima de gases extraída de la de aguas pluviales de más de 80 grados Fahrenheit que se pueden encontrar en las minas de carbón agotadas. Estas aguas circulan por una estrecha red de tuberías en las paredes del edificio de la Escuela de Gestión y Diseño Zollverein concebida por el estudio de arquitectura SANAA, y que maneja todos sus requerimientos de calefacción y refrigeración sin ningún tipo de aislamiento o suministro de energía externa.

Pero el interés también debería dirigirse a la relación urbana establecida entre los diversos complejos de edificios (o islas urbanas) en un océano de naturaleza industrial (Industrienatur). A medida que la población mundial continúa urbanizándose y el terreno disponible para el desarrollo de nuevas construcciones se vuelve cada vez más escaso, es importante entender las ideas ofrecidas por el complejo minero que lo diferencian de otros conjuntos industriales de principios del siglo XX que se insertan dentro de una tendencia contemporánea de re-utilizar edificios industriales para uso culturales o artísticos. Pero el complejo Zollverein además de haber sido transformado en conjunto cultural también sugiere una nueva urbanidad basada en islas urbanas rodeadas de Industrienatur. Dentro de una realidad en que las ciudades han pasado a ser cada vez más extensas, difusas y en las Américas de baja densidad, la estrategia sugerida por el complejo del Zollverein ofrece la posibilidad de establecer un tejido urbano de núcleos densos rodeados por un paisaje de bajo mantenimiento que tiene el potencial de unir las virtudes del suburbanismo con los de la ciudad tradicional.

\section{Notas y referencias}

1 Fritz Schupp, Martin Kremmer / con una introducción de Kurt Wilhelm-Kästner con un epílogo en la nueva publicación de W. Busch; y publicado nuevamente por Roland Jaeger. (Berlin: Gebr. Mann, c1997) Busch, W, Die Architekten Schupp \& Kremmer und ihr Beitrag zum industriellen Welterbe des 20. Jahrhunderts: die Zeche Zollverein in Essen und das Erzbergwerk Rammelsberg in Goslar https://www. icomos.org/risk/2007/pdf/Soviet Heritage_29_IV-5_Busch.pdf retrieved 11/3/2018 https://historischesportal.essen. de/historischesportal_namen/friedhof/ friedhofsfuehrer/friedhofsfuehrer detailseite_876940.de.html retrieved $11 / 3 / 2018$ 
2 J. Herf. Reactionary Modernism: Technology, Culture, and Politics in Weimar and the Third Reich / Cambridge (Cambridge Univ. Press 2003) quoted in K. James-Chakraborty, 'Inventing Industrial Culture in Essen'" in, ed. P. Jaskot and G. Rosenfeld Beyond Berlin: German Cities Confront the Nazi Past. (Ann Arbor: University of Michigan Press. 2007), pp.126

3 http://www.rheinischeindustriekultur.com/ seiten/objekte/orte/essen/objekte/bergbau/ zeche_zollverein_12_gesamtanlage. html Zeche Zollverein 12 | Gesamtanlage Essen, Bullmannaue http://www. rheinischeindustriekultur.com/seiten/ objekte/orte/essen/objekte/bergbau/zeche zollverein_3-7-10_gesamtanlage.html

4 Germany's effort to phase out and rationalize its fossil-fuel subsidies. https://www.oecd. org/site/tadffss/Germany-Peer-Review.pdf

5 https://www.open-iba.de/en/ geschichte/ 1989-1999-iba-emscher-park/

6 C. Beanland "Inside the Coalmine That Has Been Transformed from Defunct Industrial Wasteland to a Beautiful Park for Locals" The Independent. Monday 20 March 2017 https://www.independent.co.uk/travel/ europe/zollverein-german-coal-minetransformed-park-dusseldorf-germanytours-a7631231.html

7 S. Hemmings and M. Kagel. 'Memory Gardens: Aesthetic Education and Political Emancipation in the Landschaftspark Duisburg-Nord.' German Studies Review Vol. 33, No. 2 (May 2010), pp. 243-261. A. Lubov. 'The Anti-Olmstead', The New York Times Magazine'. (May 16, 2004) https:// www.nytimes.com/2004/05/16/magazine/ the-anti-olmsted.html

8 P. Latz, "Drei Annäherungen", manuscrito original fechado del, 13.11.2002 on "Industriefolgelandschaft als Aufgabe der Gartenkultur - Drei Annäherungen" in: Ml Rohde, / R. Schomann, (eds.)"Historic Gardens Today", Garden History, 33, no. 1, (2005), pp. 151. U. Weilacher Syntax of Landscape. The Landscape Architecture by Peter Latz and Partner. (Basel, Boston, Berlin. 2008), pp.102-133.

9 Alguna de la informacón en la cuál esta basado este artículo viene de una conversación entre Sascha Wienecke de Plannergruppe Oberhausen y el autor en la ciudad de Oberhausen en Junio de 2018. www.planergruppe-oberhausen.de. https:// www.open-iba.de/en/geschichte/19891999-iba-emscher-park/zeche-und-kokereizollverein-essen..

10 H.Marth,ed. Zollverein: World Heritage Site and Future Workshop, (Berlin Jovis 2018), pp. 12-15

11 J. Rusen,'Industriedenkmale und Geschichtskultur im Ruhrgebiet.'Forum Industrie-Denkmalpflege und Geschichtskultur.(1998) https://industriekultur.de/ik/2017/10/18/essen-heft-217-derzeitschrift-forum-geschichtskultur-ruhrerschienen/ T. Edensor' Industrial Ruin: Space, Aesthetics and Materiality' (Oxford: Berg, 2005), pp.126. and R. Hewison 'The Heritage Industry: Britain in a Climate of Decline' (London: Methuen 1987)

12 J. Blia, 'Criticality in Phenomenal Memory: Architectural Mnemonics for the Chaudiere'. Carleton University Ottawa, Ontario Graduate Thesis September 1, (2007), pp. 1-75. https://curve.carleton.ca/ system/files/etd/c56a1f07-9ca1-4bec-ab65682fb8b11ff8/etd_pdf/4d18142af4a329d 3e 5aa64ccbdc5acf8/blias-c-criticalityinp henomenalmemoryarchitectural.pdf) Vea también Sebastian Marot, Sub-Urbanism and the Art of Memory (London: Architectural Association. 2003), pp.24-33.

13 J. Blia, 'Criticality in Phenomenal Memory: Architectural Mnemonics for the Chaudiere'. Carleton University Ottawa, Ontario Graduate Thesis Septiembre 1, (2007), pp. 1-75. https://curve.carleton.ca/ system/files/etd/c56a1f07-9ca1-4bec-ab65682fb8b11ff8/etd_pdf/4d18142af4a329d 3e 5aa64ccbdc5acf8/blias-c-criticalityinp henomenalmemoryarchitectural.pdf) See also Sebastian Marot, Sub-Urbanism and the Art of Memory (London: Architectural Association. 2003), pp.24-33.

14 M.Treib, "An Industrial Sublime" en Peter Latz, Rust Red Landscape Park DuisburgNord. (Munich: Hirmer, 2016), pp. 9-11.

15 F. Ankersmit Sublime Historical Experience (Stanford: Stanford University Press, 2005) https://www.scribd.com/doc/159563604/ 
Whitechapel-Documents-of-ContemporaryArt-Simon-Morley-Editor-The-SublimeWhitechapel-Documents-of-ContemporaryArt-The-MIT-Press-2010

16 P. Shaw, The Sublime London( Routledge, 2006), pp.7 and Simon Morley Introduction// The Contemporary Sublimesimonmorley. com/biography/The_Sublime-An Introduction.pdf

17 P. Latz. 'Drei Annäherungen', manuscrito original fechado del13.11.2002 on "Industriefolgelandschaft als Aufgabe der Gartenkultur - Drei Annäherungen Leipzig (2003), pp. 63 U. Weilacher: Syntax of Landscape: The Landscape Architecture of Peter Latz and Partners. (Basel: Birkhäuser, 2008), pp. 102-133.

18 C.F. Schröer, 'Paradies mit Promenade = Paradise with Promenade', Topos: European Landscape Magazine Iss.41 (December, 2002), pp. 115-118.

19 P. Zucker, 'Space and Movement in High Baroque City Planning.: Journal of the Society of Architectural Historians, Vol. 14, No. 1 (Mar. 1955), pp.8-13, Christian Norbert Schultz, Baroque Architecture. (Milano: Electa, 1971), pp. 19-61. S., Kostof. The City Shaped Urban; Patterns and Meaning through History. (London: Thames and Hudson, 1991), pp 209-276. C.F. Schröer, 'Paradies mit Promenade $=$ Paradise with Promenade'I, Topos: European Landscape Magazine Iss.41 (December, 2002), pp. 115118.

20 PDF) Duisburg-Nord. Available from: https://www.researchgate.net/ publication/309375364_Duisburg-Nord [accessed Aug 30 2018].

21 Información sobre las ideas del archipiélago de Matias Ungers se pueden encontrar en P.V. Aureli. The Possibility of an Absolute Architecture. (Cambridge: MIT Press, 2014), Karen Lohrmann, The City in the CityBerlin: A Green Archipelago, (Zuerich:Lars Mueller Publishers, 2013 ). J.Walker Islandsin-the-City: Berlin's Urban Fragments, The Journal of Architecture, 20:4, (2015).y en la revista Lotus International No. 19, Junio (1978) 